\title{
Digital Disruption of Dietetics: are we ready?
}

\begin{abstract}
Digital health is transforming the delivery of health care around the world to meet the growing challenges presented by ageing populations with multiple chronic conditions. Digital health technologies can support the delivery of personalised nutrition care through the standardised Nutrition Care Process (NCP) by using personal data and technologysupported delivery modalities. The digital disruption of traditional dietetic services is occurring across the world, supporting responsive and high-quality nutrition care. These disruptive technologies include integrated electronic and personal health records, mobile apps, wearables, artificial intelligence and machine learning, conversation agents, chatbots and social robots. In this paper, we outline how digital health is disrupting the traditional model of nutrition care delivery and outline the potential for dietitians to not only embrace digital disruption but take ownership in shaping it, in order to enhance patient care. This paper provides an overview of digital health concepts and disruptive technologies according to the four steps in the NCP, nutrition assessment, diagnosis, intervention, and monitoring and evaluation. It is imperative that dietitians stay abreast of these technological developments and be the leaders of the disruption, not simply subject to it. By doing so, dietitians now, and in the future, will maximise their impact and continue to champion evidence-based nutrition practice.
\end{abstract}

Keywords: Diet, Nutrition Care Process, Digital Health, Wearables, Telehealth, Mobile Health 


\section{Introduction}

Seventeenth century German scientist Georg Christoph Lichtenberg is famous for his proclamation, "I cannot say whether things will get better if we change; what I can say is they must change if they are to get better". This assertion has never been more relevant for today's health systems in managing the complications of a rapidly ageing global population with multimorbidity [1]. With the majority of disease burden attributable to modifiable lifestyle factors, such as poor diet and physical inactivity [1], health and social care services face a considerable challenge in how to meet medical, lifestyle and personal health needs of populations they serve. A daunting challenge for health care managers is how to provide the required care in a scalable, clinical and cost-effective way, within finite budgets. One strategy to mitigate burden on health care systems has been to empower individuals to selfmanage their health, through health system and service delivery innovations [2].

Digital health has been around for the last two decades in the form of medical informatics and simple telehealth, but it's only in the past five years that there has been a proliferation of literature on this topic [3]. Digital health employs routine and innovative forms of information and communications technology to address health needs and remotely deliver effective health interventions [4]. Digital health includes electronic health (eHealth), such as web-based and software programs; mobile health (mHealth) such as smartphone applications (apps), text messaging programs and wearable devices; health information technology; telehealth/telemedicine; electronic medical records (EMRs) and emerging areas such as the use of advanced computing sciences in big data, genomics and artificial intelligence [4]. Digitally enabled systems, that is, health systems that are adopting 
technology to help improve the quality, delivery and management of patient care, have the potential to transition our healthcare model to harmonise primary and secondary care with self-management as the central pillar.

In this paper, we outline how digital technologies are disrupting the traditional delivery of nutrition care and how dietitians can embrace the digital world to support delivery of the Nutrition Care Process (NCP).

\section{Dietetics in the Digital World}

It has been two decades since the Journal of Human Nutrition and Dietetics published 'Dietitians and the internet: are dietitians embracing the new technology?' [5]. It provided fascinating insights and a yardstick to the subsequent digital disruption that has occurred since. The authors found only $66 \%$ of dietitians had internet access in the workplace and perhaps even more tellingly, only $13 \%$ of dietitians reported seeing information obtained by patients from the internet [5]. Fast-forward twenty years and we are not discussing if dietitians are using 'new' technology, but how dietitians can shape the development and implementation of technologies to leverage their expertise and improve patient outcomes [6]. The recent British Dietetic Association (BDA) workforce strategy for Dietetics 20202030 calls on dietitians to embrace advances in science and technology alongside a growing recognition that this will re-define how dietitians develop and deliver medical nutrition therapy (MNT) [7]. Ubiquitous and almost limitless access to information is transforming dietetic roles from being gatekeepers of nutrition knowledge to facilitating and motivating patients to access and implement scientifically sound, evidence-based care. Technology has advanced exponentially over the last 20 years, with cost and barriers to access dropping 
considerably $[8,9]$, resulting in dietitians and patients now surrounded by an increasingly digital, highly connected world. From increasing connectivity to 5G wireless networks, portable smartphone devices with various applications, integrated EMRs, and telehealth increasing complexity brings opportunities for dietetics to innovate, to support patients in managing their own nutrition (see Figure 1 for an overview of digital technologies that can complement the NCP).

The proliferation of available technologies for healthcare delivery in recent years continues to expand in the context of COVID-19 and its immediate impact on dietetic practice. Some innovations showcase the potential for dietetic practice to be improved via digital health. For example, since 2017, dietitians in the United Kingdom (UK) have had access to free, on-demand webinars (www.patientwebinars.co.uk) for patients, as part of their treatment and/or education on specific gastroenterology conditions [10]. In the past 6 months, this resource has been used across the UK by primary care practices and dietetic departments. Furthermore, during the shutdown of traditional service delivery due to COVID-19, dietitians worldwide have turned to telephone and video consultations to deliver high quality nutrition care, services that are comparable to face-to-face delivery modalities [11]. This rapid adoption of virtual care has been supported by policy change in many jurisdictions, to pave the way for a future where this practice is commonplace [12].

Despite these recent advancements, the literature indicates adoption of technology into health care practice has been relatively slow in recent years. While dietitians typically use face-to-face consultations, patients may be more interested in technology-assisted consultations [13]. A 2019 survey by Abrahams et al. [14] demonstrated that most dietitians did not consider technology to play an important role in their current practice. This may be 
due to a lack of e-readiness [15], training and professional development, remuneration, or exposure to the opportunities it provides to enhance efficiency and delivery of patientcentred care [11].

This year, an editorial in this Journal clearly outlined that dietitians who do not adopt or understand the transforming digital dietetics landscape run the risk of being replaced [6]. It is therefore paramount that dietitians be the leaders of this digital disruption, rather than be subject to it. We believe this is a critical time in our profession to ensure dietetic services remain relevant and supported in digital healthcare futures, whilst ensuring practice is underpinned by a robust evidence base.

\section{Digital health records and the NCP}

EMR is a digital form of patient records that allows for both current and historic assessment information to be collected seamlessly [16] and have prove efficient in the documentation of administrative and clinical processes for healthcare purposes. EMRs result in improved dietitian access to nutrition information, increased numbers of resolved nutrition diagnoses and enhanced capacity and efficiency of dietetic services [17].

Despite increasing use of electronic, internet-enabled records (of over $50 \%$ annually since 2013 [18]), coordination gaps remain such as lack of interoperability across multiple digital health platforms and systems, and technical barriers experienced by clinicians [19, 20]. The growing adoption of personal health records is assisting to address this problem, giving patients the ability to access, manage and share their health information, and that of others for whom they are authorised, in a private, secure and confidential manner [21]. Personal 
health records differ from standard EMRs and have already been implemented in many countries, including Australia, Austria, Denmark, Estonia, Finland, France, Norway and Sweden; and in the United States are variably offered through private health providers [21].

\section{Digitally Disrupting Nutrition Assessment}

Digital health lends itself well to enhancing nutrition assessment by leveraging the plethora of technologies on the market, including smartphone apps, sensors and wearables, together with drawing relevant data from EMRs and cloud computing systems.

Chen and colleagues [22] highlight how dietitians can make use of mobile apps in nutrition assessment; for example, to track patients' body weight and/or blood glucose levels, their activity through a pedometer and exercise diary, and for documenting dietary intake [22]. Key conclusions from the review highlighted that mobile apps have great potential to complement nutrition care delivered by dietitians and enhance efficiency of the nutrition care process, allowing more time for counselling patients [22]. The Pt-Global app is a nutrition assessment app that facilitates simple and systematic nutrition risk screening using the validated patient-generated subjective global assessment (PG-SGA) for interventional triaging, and for nutritional monitoring during and after intervention [23]. However, nutrition-related apps must be utilised correctly (i.e. users record data accurately) and contain evidence-based algorithms to be effective, so are not considered appropriate for standalone use. But, when combined with dietitian prescription, critical thinking and monitoring, these apps may support individualised, patient-centred nutrition care [24]. 
Wearable devices, defined as infrastructures that interconnect technology with wearable sensors through wireless connections [25], offer a simple and non-invasive mechanism to inform nutrition assessment. Wearables typically sync with a software system or mobile app that can be used to digitally exchange nutrition assessment parameters. Examples of wearables that may assist nutrition assessment are summarised in Figure 1 and include: wireless tracking of body weight (via Bluetooth connected scales or weight tracking mobile apps) [26], continuous glucose monitors and smart insulin pens (that track dose/time and recommend correct insulin type/dosage) [27], smartwatches (e.g. Apple watch) and activity monitors (e.g. Fitbits; tracking activity, heart rate, sleep patterns), vital sign patches (that wirelessly track and monitor patients' heart rate, respiration, temperature, steps taken, sleep cycle, stress levels, and whether a user has fallen or otherwise become incapacitated [28]), wireless electrocardiogram monitors [29] and wearable blood pressure monitors [30].

Technology-based methods for dietary intake assessment have also been systematically evaluated. A review found that one-third of such tools used image-based methods and twothirds used integrated databases to estimate energy or nutrient intakes using predominantly self-reported data [31]. In addition to mobile apps to assess dietary intake [24], technologyassisted dietary assessment includes web-based methods in individuals with and without disabilities [32] and automated electronic methods among low income adults [33]. Some devices have been proposed to automatically scan and track dietary intake [34] and analyse food diaries for their nutrient profiles using machine learning algorithms, by photographing meals [35]. One novel hybrid dietary assessment tool currently under development (VoiceImage-Sensor technologies for Individual Dietary Assessment; VISIDA) uses an image- 
voice food record app, wrist sensor, web-based analysis platform and machine learning models, and will undergo iterative development and piloting in low income countries [36].

The validity of apps for nutrition assessment is important for dietitians to consider. Studies have shown that dietary assessments conducted through mobile phones and nutrition apps have similar validity and reliability $[37,38]$. Pedometers and activity trackers can reliably collect activity measures for structured ambulatory activity; however, have low validity for predicting energy expenditure [39]. In contrast, the accuracy, clinical validity and quality of mobile health apps for blood glucose monitoring in diabetes management require further scrutiny [40]. It is important to acknowledge that many of the available commercial health apps may not be validated and are not endorsed by reputable authorities [41], and seldom include evidence-based behaviour change techniques [42].

It is important for dietitians to understand the availability and benefits of these technologyassisted nutrition assessment tools. Main barriers to virtual nutrition care delivery include difficulties in taking accurate anthropometric measurements and identifying clinical signs of nutrient deficiencies [43]. The imperative for dietitians to be abreast of such technology is recognised by professional agencies such as the BDA via strategic partnerships [44] to assist dietitians in understanding how to leverage digital health to support and improve their practice [6]. Libraries of apps are also becoming widely available to support dietitians in providing evidence-based digital resources and suggestions for devices and mobile apps in their practice (Table 1). If dietitians require patients to collect nutrition assessment data using technology, they should also engage with patients prior to the consultation to outline what technology needs to be downloaded or installed. Next, patients should be shown how 
to record anthropometric measurements using home-scales, pedometers and wearables; or told how to request such measurements to be taken by other practitioners working in the patient's location.

\section{Digitally Disrupting Nutrition Diagnosis}

Many programs and computer software platforms exist for guiding dietitians in making an appropriate nutrition diagnosis, as recently overviewed by Chen and colleagues [22]. Some promising technologies being used to inform nutrition diagnosis are artificial intelligence (AI) and deep machine learning. AI has the capability to read EMR data via text recognition with natural language processing, including medical history, medications, and results from physical assessments, imaging and pathology; and contextualise it to generate diagnosis and/or treatment decisions and possibilities [45]. There have been calls for the development of algorithm-driven decision-aid technology in nutrition, for example, in predicting the risk of malnutrition using blood biomarkers [46]; however, despite great promise in dietetics, there are limited examples to date of $\mathrm{AI}$ and deep learning being used to predict nutrition diagnoses.

We only need to look at the uptake and application of AI and deep learning in the wider medical literature to find examples of traditional human tasks being automated by computer-driven AI. Recent studies show how elements of chronic disease management can be improved through AI; for example, one has demonstrated that deep learning of EMR data can predict risk of disease progression, future medical outcomes and care decisions in diabetes and mental health [47]. Such technology could be used to streamline nutrition 
diagnosis by collating information already captured in EMRs such as anthropometric, biochemical, clinical and nutrition care data, and using it to predict nutrition diagnoses.

\section{Digitally Disrupting Nutrition Intervention}

Effective nutrition interventions rely on individual behaviour change. The principles of long-term behaviour change and maintenance are well recognised and include motivation, self-regulation, access to resources (both psychological and physical), habit formation, and environmental and social influences [48]. Technology-assisted dietetic services, including telephone/video consultations, on-demand webinars, mHealth and eHealth are intervention delivery modalities particularly useful for facilitating behaviour change and improving chronic disease self-management [49-53] and are slowly seeing uptake in clinical systems $[54,55]$. Text-message programs delivered by dietitians are shown effective at improving dietary behaviour in people with cardiovascular disease [56, 57], and web-based nutrition interventions have been shown to improve dietary behaviour among patients with obesity [58] and type 2 diabetes [59]. Similarly, community-based dietetic mHealth and eHealth interventions have demonstrated the capacity to improve dietary behaviour in single studies among people with type 2 diabetes [60], hypertension [61], chronic kidney disease [62] and eating disorders [63]. While these interventions have the potential to improve a variety of health-related behaviours both in hospital- and community-based dietetic services, it is important to note there are a similar number of studies suggesting these interventions may not be effective [64-67]. Hence, larger and more robust clinical trials are needed to confirm the clinical benefits of these methods in practice before integration into usual care systems. 
The ubiquitous nature of technology means that consumers have greater access to digital resources than ever before [68]. A 2015 survey demonstrated 58\% of smartphone users had downloaded a health-related app for their lifestyle self-management [69]. Natural language processing, AI and machine learning have also driven increased availability of point-ofcare health information such as chatbots, which can provide lifestyle and medical advice through conversational interactions with the user. There are many established AI chatbots commercially available, for example, Woebot, Your.MD, Babylon and HealthTap, where patients can input their symptoms and advice is generated instantly [70]. Nutrition-specific examples are emerging too, for example, Health Hero [71], Tasteful Bot [72] Lark [73] and Forksy [74], which all provide tailored nutrition advice using AI algorithms via Facebook messenger or smartphone app platforms.

Smart voice assistants or conversation agents, like Amazon Alexa and Google Home, are being increasingly used to support people at home via AI-driven conversations. Interactive conversations with smart voice assistants may enable patient-centred and patient-engaged approaches to care, which can empower and motivate patients to take more control of their health [75]. A limited number of trials have evaluated smart voice assistants for behaviour change purposes. A systematic review of conversational agents in healthcare showed these agents are commonly used for both patient support (providing education and training for health-related aspects of their lives) and clinician support (used to autonomously conduct clinical interviews with diagnostic purposes in mental health and sleep disorders; and assist with data collection and decision support in referral management) [75]. One randomised controlled trial found that a conversational agent improved targeted health behaviours, including fruit and vegetable intake ( +3 serves per day), compared to a control group [76]. 
Another study evaluated an automated and interactive telephone program designed by dietitians to improve DASH-diet adherence among African Americans with hypertension. Significant improvements in diet quality, fibre intake and daily energy expenditure were found, along with trends towards reductions in blood pressure and improved medication adherence, however no differences were observed between intervention and control groups and uptake was modest (15\%) [77]. This highlights the importance of including end-users in the design of technology assisted models of care.

In the not too distant future, $\mathrm{AI}$ and machine learning may be used to conduct rapid quality improvement projects and analyse outcome data captured by dietitians through NCP terminologies. Hypothetically, variations in nutrition interventions used in standard clinical practice could be evaluated in real time, with potential for algorithms to inform recommendations for the most appropriate nutrition intervention for each clinical case. This is an example of a move towards precision nutrition, which can further personalise recommendations [45] while supporting dietitians in complex clinical decision making via electronic decision pathways [78].

\section{Digitally Disrupting Nutrition Monitoring and Evaluation}

Routine self-monitoring encourages self-efficacy and motivation and is therefore important for long-term behaviour change. Digital health has the potential to improve self-monitoring behaviours by collecting live health information that can facilitate remote reviewing and monitoring. Similarly, the implementation and adoption of personal health records allows individuals to share health data with their dietitian, which can be used to track and evaluate nutrition- and health-related goals. Mobile apps are another efficient way to track diet and 
health data, with over $60 \%$ of dietitians working in various practice areas (including both private practice and hospital) using mobile apps with their clients to record food intake and track progress [79-81]. Digital health also offers the opportunity to facilitate collection of patient-reported outcome and experience measures (PROMs/PREMs) [82], which can be used to enhance nutrition review and evaluation [83]. As automation and computer systems advance, PROM and PREM data may disrupt the NCP further. For example, electronic patient-reported data have potential to flag patients needing further support or monitoring, and additional intervention modification may be possible through AI and deep learning based on PROMs and PREMs inputs recorded in the system.

Digital tools can allow dietitians to remotely monitor and evaluate nutrition care outcomes and advance nutrition research and evidence-based practice. One example is the Academy of Nutrition and Dietetics Health Informatics Infrastructure (ANDHII) [84]. ANDHII is an electronic web-based tool that could potentially enable efficient evaluation of NCP chains in large datasets. A feasibility study showed that dietitians could use the platform with negligible impact on their practice time, whilst ensuring evidence-informed practice [85]. Wider adoption of platforms with similar capabilities as ANDHII will continue to occur, saving time and automating many tasks involved in monitoring and evaluating the NCP. Other technologies to engage patients in monitoring their own nutrition are also emerging, such as a program that allows patients to track their dietary intake alongside their individual nutrition requirements from the hospital bedside [86]. This not only encourages patientcentred and patient-driven care, but could enable finite dietitian time to be directed to more high value clinical activities by allowing patients to conduct simple tasks themselves (e.g. recording their own dietary intake). Future nutrition monitoring and evaluation modalities 
may involve deep machine learning and/or AI algorithms to detect appropriate monitoring parameters in EMR and generate suggested review times. As dietitians embrace these technologies, we are likely to see more effective use of dietitian time and the efficient evaluation of nutrition interventions, supporting the advocacy for workforce funding and technology-supported models of care.

\section{Digital Dietetic Frameworks and Resources}

Nutrition care supported by mHealth appears to be a highly accepted, safe and effective way to support self-management among people with chronic conditions [51, 52], causing some groups to recommend wider adoption of digital nutrition interventions. Numerous online databases, resources and frameworks are available to support health professionals to select, implement and evaluate mHealth technologies (as summarised in Table 1).

The active role of dietitians will never be lost to machines; however, the dietetic profession will need to adapt to these digital education delivery platforms in ways that may challenge conventional service delivery. Just as it is dietitians' responsibility to maintain contemporary knowledge on clinical evidence in their area of practice, dietitians will be increasingly required to be aware of available technology and the evidence underpinning its use. Organizations such as ORCHA [87] and the NHS's Apps Library [88] are valuable, for dietitians to access knowledge of what is available and evidence-based (as detailed in Table 1). Professional organisations also recognise their critical role in ensuring dietitians stay abreast of current technology, and are making resources and training more available; for example, practice guides, webinars and resources [89-91] and position statements [11]. If dietetic professionals stay up to date with this growing evidence base, future 
interventions may well include provision of evidence-based digital resources, devices and mobile apps.

Tertiary programs must consider the professional training the next generation of dietitians need in order to be equipped with the skills and confidence to deliver high quality nutrition care using technology in the era of digitally disrupted healthcare systems. Appropriate and effective use of technology in practice is a key competency outlined in many international dietetics training standards, including Australia [92], Canada [93], and the United Kingdom [94]. These standards also emphasise the need for education on the use of digital health technologies for patient education and counselling, and throughout other important areas of professional practice. Integrating digital heath skills in undergraduate dietetic teaching, or as continuing professional development, has been shown to improve understanding of concepts essential for using technology-supported mediums [95].

\section{Barriers: Equity, data security, software and policy considerations}

Although technology presents endless opportunities for the dietetic profession, we must be aware of inherent inequities that may occur. Such challenges include health care systems' budgets and whether individuals can afford/have access to technology hardware or reliable phone/internet services due to financial disadvantage. For example, it may not be appropriate for a dietitian to ask a patient to purchase a wearable, or download an app, if they cannot afford one or have limited mobile/internet usage allowances. This highlights the need for carefully considered research into telehealth tools and technology-supported models of care, to consider the advantages and disadvantages of minority groups (whom generally lack representation in telehealth research), ensure equal access, and overcome 
literacy barriers to engaging underserved communities, otherwise there will be a significant danger of perpetuating or escalating current disparities [96]. An important environmental enabler of digital health implementation is policy support. Many countries have digital health policies in place, or are in the process of developing them; for example, Australia, China, France, Germany, India, Indonesia, Japan, Korea, Malaysia, Philippines, Singapore, Sweden, Thailand, the European Union, the United States and Vietnam currently have relevant policies in place for eHealth and/or the Internet of Things for healthcare [97, 98]. The UK created a digital health action framework in 2014 to support frontline staff, patients and citizens to take better advantage of digital health technologies in practice [99], and has since seen a number of supporting policies [100]. The World Health Organization reports over $66 \%$ of countries have at least one health information system policy, $58 \%$ have an eHealth policy or implementation strategy and over $25 \%$ have policies for telehealth [101]. In addition, the World Health Organization keeps an updated directory of relevant digital health policies for each country in its online database [102]. Digital health applications that support clinical decision-making processes often require medical device approval [103] and strong policy surrounding these approvals is required. As such, resources are available in certain countries to support developers in designing digital devices that meet regulations $[104,105]$.

Implementing digital health services is only possible when widely accepted and adopted by consumers, clinicians and health systems collectively. One of the biggest barriers to this scaled implementation is concerns around data safety and confidentiality. For example, one 2016 study showed that many mobile apps' manifest file privacy declarations did not match those in the source code, raising questions around app quality and highlighting potentially 
negative impacts on the safety and reliability of mHealth-related applications [106]. Further questions exist on who owns, can view and controls health data stored on cloud services, suggesting the use of digital health systems requires careful strategic planning and transparent guidelines.

The interoperability of digital health devices and systems presents another threat to wider adoption of digital health services. Semantic interoperability refers to the ability for digital devices to communicate and exchange information with each other, which is necessary for the sharing of nutrition tracking information with EMRs and big data techniques to support decision-making processes [107]. There has been significant effort in recent years to introduce international standards across health technology platforms, with organisations such as Health Level Seven (HL7) International leading the way with the Fast Health Interoperability Resources (FHIR) [108]. FHIR aims to accelerate the interoperability between EMRs and mHealth solutions in order to increase access to health data for large scale analytics [109].

Finally, remuneration for dietetic services delivered via technology has historically been challenging for dietitians and the wider health community $[110,111]$. Many countries such as Australia, Canada, China, Italy, UK and United States [12, 112-115] have recently (and temporarily) adopted remuneration policies around virtual care (telehealth) in response to COVID-19. These efforts to reduce barriers to telehealth implementation underscore the potential to reframe traditional models of dietetic interventions into virtual and distance modalities [12]. Future evaluations will give insight into the long-term policy decisions of telehealth-delivered dietetic services. However, these policies do not include asynchronous 
digital health modalities (one-way communication at any time; e.g. text-messaging and web-portals), which means these services still require fee-for-service models in practice. It is time for decision makers and private health payers to strongly consider the evidence for expanding telehealth to include mHealth and eHealth. These technologies can be used alongside telephone or video conferencing modalities or in-person delivery as they improve access to effective nutrition services and support people with chronic conditions to optimise their diet-related health and well-being, regardless of their location, income or literacy level.

\section{Conclusion}

There are many examples of effective technology-supported dietetic programs that can enhance the delivery of medical nutrition therapy as part of the Nutrition and Dietetic Care Process, to improve dietary and clinical outcomes for patients and populations. In order to achieve widespread adoption of digital nutrition care interventions, issues of data security, policies and regulations, interoperability and remuneration require attention. The digital disruption of traditional NCP delivery is here, and this paper calls on dietitians around the world to be the leaders of this disruption, not the subject of it. It is imperative that dietitians stay abreast of technological developments and understand how digital health can be used to support and improve their current practice, to ensure dietitians remain effective and are the champions of evidence-based nutrition care.

\section{Conflicts of Interest}

The authors have no conflict of interest to declare. 
References

1. James SL, Abate D, Abate KH, Abay SM, Abbafati C, Abbasi N, Abbastabar H, Abd-Allah F, Abdela J, Abdelalim A: Global, regional, and national incidence, prevalence, and years lived with disability for 354 diseases and injuries for 195 countries and territories, 1990-2017: a systematic analysis for the Global Burden of Disease Study 2017. The Lancet 2018, 392(10159):1789-1858.

2. Mitchell M, Kan L: Digital Technology and the Future of Health Systems. Health Systems \& Reform 2019:1-8.

3. Ahmadvand A, Kavanagh D, Clark M, Drennan J, Nissen L: Trends and visibility of "digital health" as a keyword in articles by JMIR publications in the new millennium: Bibliographic-bibliometric analysis. Journal of medical Internet research 2019, 21(12):e10477.

4. Organization WH: WHO guideline: recommendations on digital interventions for health system strengthening: web supplement 2: summary of findings and GRADE tables. In.: World Health Organization; 2019.

5. Kirk S, Cade J, Greenhalgh A: Dietitians and the internet: are dietitians embracing the new technology? Journal of human nutrition and dietetics 2001, 14(6):477-484.

6. Abrahams M, Matusheski N: Personalised nutrition technologies: a new paradigm for dietetic practice and training in a digital transformation era. Journal of Human Nutrition and Dietetics 2020, 33(3):295-298.

7. Hickson M, Child J, Collinson A: Future Dietitian 2025: informing the development of a workforce strategy for dietetics. Journal of human nutrition and dietetics 2018, 31(1):23-32.

8. Kvedar J, Coye MJ, Everett W: Connected health: a review of technologies and strategies to improve patient care with telemedicine and telehealth. Health Affairs 2014, 33(2):194-199.

9. Tuckson RV, Edmunds M, Hodgkins ML: Telehealth. New England Journal of Medicine 2017, 377(16):1585-1592.

10. Williams M, Barclay Y, Harper L, Marchant C, Seamark L, Hickson M: Feasibility, acceptability and cost efficiency of using webinars to deliver first - line patient education for people with Irritable Bowel Syndrome as part of a dietetic - led gastroenterology service in primary care. Journal of

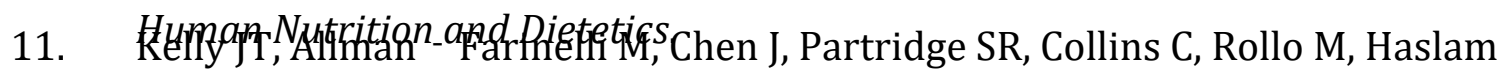
R, Diversi T, Campbell KL: Dietitians Australia position statement on telehealth. Nutrition \& Dietetics 2020.

12. Torous \}, Jän Myrick K, Rauseo-Ricupero N, Firth J: Digital Mental Health and COVID-19: Using Technology Today to Accelerate the Curve on Access and Quality Tomorrow. JMIR mental health 2020, 7(3):e18848.

13. Elvin - Walsh L, Ferguson M, Collins PF: Nutritional monitoring of patients post - bariatric surgery: implications for smartphone applications. Journal of human nutrition and dietetics 2018, 31(1):141-148. 
14. Abrahams M, Frewer LJ, Bryant E, Stewart-Knox B: Personalised nutrition technologies and innovations: A cross-national survey of registered dietitians. Public Health Genomics 2019, 22(3-4):119-131.

15. Van Dyk L: A review of telehealth service implementation frameworks. International journal of environmental research and public health 2014, 11(2):1279-1298.

16. Kruse CS, Stein A, Thomas H, Kaur H: The use of electronic health records to support population health: a systematic review of the literature. Journal of medical systems 2018, 42(11):214.

17. McCamley J, Vivanti A, Edirippulige S: Dietetics in the digital age: The impact of an electronic medical record on a tertiary hospital dietetic department. Nutrition \& Dietetics 2019, 76(4):480-485.

18. Office of the National Coordinator for Health Information Technology: Officebased Physician Electronic Patient Engagement Capabilities. In.: The Office of the National Cordinator for Health Information Technology 2016.

19. Cimino JJ: Improving the electronic health record-are clinicians getting what they wished for? Jama 2013, 309(10):991-992.

20. Evans RS: Electronic Health Records: Then, Now, and in the Future. Yearbook of medical informatics 2016, Suppl 1(Suppl 1):S48-S61.

21. Australia Digital Health Agency: Digital Health Evidence Review. In.: Australian Government, Canberra. Available at https://www.digitalhealth.gov.au/get-started-with-digital-health/digitalhealth-evidence-review.

22. Chen J, Gemming L, Hanning R, Allman-Farinelli M: Smartphone apps and the nutrition care process: Current perspectives and future considerations. Patient education and counseling 2018, 101(4):750-757.

23. Jager - Wittenaar H, de Bats HF, Welink - Lamberts BJ, Gort - van Dijk D, van der Laan BF, Ottery FD, Roodenburg JL: Self - completion of the patient generated subjective global assessment short form is feasible and is associated with increased awareness on malnutrition risk in patients with head and neck cancer. Nutrition in clinical practice 2020, 35(2):353-

24. $\quad$ $62 \mathrm{n}$ J, Gemming L, Hanning R, Allman-Farinelli M: Smartphone apps and the nutrition care process: Current perspectives and future considerations. Patient education and counseling 2018, 101(4):750-757.

25. Bhatt $Y$, Bhatt C: Internet of things in healthcare. In: Internet of things and big data technologies for next generation HealthCare. edn.: Springer; 2017: 13-33.

26. Chen J, Cade JE, Allman-Farinelli M: The most popular smartphone apps for weight loss: a quality assessment. JMIR mHealth and uHealth 2015, 3(4):e104.

27. Sangave NA, Aungst TD, Patel DK: Smart Connected Insulin Pens, Caps, and Attachments: A Review of the Future of Diabetes Technology. Diabetes Spectrum 2019:ds180069. 
28. Levine DM, Ouchi K, Blanchfield B, Diamond K, Licurse A, Pu CT, Schnipper JL: Hospital-Level Care at Home for Acutely Ill Adults: a Pilot Randomized Controlled Trial. J Gen Intern Med 2018, 33(5).

29. Majumder S, Chen L, Marinov O, Chen C-H, Mondal T, Deen MJ: Noncontact wearable wireless ECG systems for long-term monitoring. IEEE reviews in biomedical engineering 2018, 11:306-321.

30. Kakria P, Tripathi N, Kitipawang P: A real-time health monitoring system for remote cardiac patients using smartphone and wearable sensors. International journal of telemedicine and applications 2015, 2015:8.

31. Eldridge AL, Piernas C, Illner A-K, Gibney MJ, Gurinović MA, De Vries JH, Cade JE: Evaluation of new technology-based tools for dietary intake assessment-An ilsi europe dietary intake and exposure task force evaluation. Nutrients 2019, 11(1):55.

32. Polfuss M, Moosreiner A, Boushey CJ, Delp EJ, Zhu F: Technology-based dietary assessment in youth with and without developmental disabilities. Nutrients 2018, 10(10):1482.

33. Kupis J, Johnson S, Hallihan G, Olstad DL: Assessing the usability of the automated self-administered dietary assessment tool (asa24) among low-income adults. Nutrients 2019, 11(1):132.

34. Torkamani A, Andersen KG, Steinhubl SR, Topol EJ: High-definition medicine. Cell 2017, 170(5):828-843.

35. Meyers A, Johnston N, Rathod V, Korattikara A, Gorban A, Silberman N, Guadarrama S, Papandreou G, Huang J, Murphy KP: Im2Calories: towards an automated mobile vision food diary. In: Proceedings of the IEEE International Conference on Computer Vision: 2015; 2015: 1233-1241.

36. VISIDA Project: Project Overview [https://www.visida.org/]

37. Nour M, Chen J, Allman-Farinelli M: Efficacy and external validity of electronic and mobile phone-based interventions promoting vegetable intake in young adults: systematic review and meta-analysis. Journal of medical Internet research 2016, 18(4):e58.

38. Sharp DB, Allman-Farinelli M: Feasibility and validity of mobile phones to assess dietary intake. Nutrition 2014, 30(11-12):1257-1266.

39. Evenson KR, Goto MM, Furberg RD: Systematic review of the validity and reliability of consumer-wearable activity trackers. International Journal of Behavioral Nutrition and Physical Activity 2015, 12(1):159.

40. Fleming GA, Petrie JR, Bergenstal RM, Holl RW, Peters AL, Heinemann L: Diabetes digital app technology: benefits, challenges, and recommendations. A consensus report by the European Association for the Study of Diabetes (EASD) and the American Diabetes Association (ADA) Diabetes Technology Working Group. Diabetes care 2020, 43(1):250-260.

41. Scott IA, Scuffham P, Gupta D, Harch TM, Borchi J, Richards B: Going digital: a narrative overview of the effects, quality and utility of mobile apps in chronic disease self-management. Australian Health Review 2018. 
42. Hoppe CD, Cade JE, Carter M: An evaluation of diabetes targeted apps for Android smartphone in relation to behaviour change techniques. Journal of human nutrition and dietetics 2017, 30(3):326-338.

43. Rollo ME, Hutchesson MJ, Burrows TL, Krukowski RA, Harvey JR, Hoggle LB, Collins CE: Video Consultations and Virtual Nutrition Care for Weight Management. J Acad Nutr Diet 2015, 115(8):1213-1225.

44. ORCHA: The Association of UK Dietitians Launch App Library to Help People Access Wellbeing Help During Covid-19 [https://www.orcha.co.uk/news/the-association-of-uk-dietitians-launchapp-library-to-help-people-access-wellbeing-help-during-covid-19/]

45. Verma M, Hontecillas R, Tubau-Juni N, Abedi V, Bassaganya-Riera J: Challenges in personalized nutrition and health. Frontiers in nutrition 2018, 5:117.

46. Zhang Z, Pereira SL, Luo M, Matheson EM: Evaluation of blood biomarkers associated with risk of malnutrition in older adults: a systematic review and meta-analysis. Nutrients 2017, 9(8):829.

47. Pham T, Tran T, Phung D, Venkatesh S: Predicting healthcare trajectories from medical records: A deep learning approach. J Biomed Inform 2017, 69:218-229.

48. Kwasnicka D, Dombrowski SU, White M, Sniehotta F: Theoretical explanations for maintenance of behaviour change: a systematic review of behaviour theories. Health psychology review 2016, 10(3):277-296.

49. Maunder K, Walton K, Williams P, Ferguson M, Beck E: eHealth readiness of dietitians. J Hum Nutr Diet 2018, 31(4):573-583.

50. Chen J, Lieffers J, Bauman A, Hanning R, Allman - Farinelli M: The use of smartphone health apps and other mobile $h$ ealth (mHealth) technologies in dietetic practice: a three country study. J Hum Nutr Diet 2017, 30(4):439-452.

51. Beratarrechea A, Lee AG, Willner JM, Jahangir E, Ciapponi A, Rubinstein A: The impact of mobile health interventions on chronic disease outcomes in developing countries: a systematic review. Telemedicine and e-Health 2014, 20(1):75-82.

52. Burke LE, Ma JZ, Azar KMJ, Bennett GG, Peterson ED, Zheng Y, Riley W, Stephens J, Shah SH, Suffoletto B: Current science on consumer use of mobile health for cardiovascular disease prevention a scientific statement from the American Heart Association. Circulation 2015:CIR. 0000000000000232.

53. Kelly J, Reidlinger D, Hoffman T, Campbell K: Telehealth methods to deliver dietary interventions in adults with chronic disease: a systematic review and meta-analysis. Am J Clin Nutr 2016, 104(6):1693-1702.

54. Goode A, Reeves M, Owen N, Eakin E: Results from the dissemination of an evidence-based telephone-delivered intervention for healthy lifestyle and weight loss: the Optimal Health Program. Translational behavioral medicine 2013, 3(4):340-350.

55. Khanal S, Lloyd B, Rissel Cs, Portors C, Grunseit A, Indig D, Ibrahim I, McElduff S: Evaluation of the implementation of Get Healthy at Work, a 
workplace health promotion program in New South Wales, Australia. Health Promotion Journal of Australia 2016, 27(3):243-250.

56. Akhu-Zaheya LM, Wa'ed YS: The effect of short message system (SMS) reminder on adherence to a healthy diet, medication, and cessation of smoking among adult patients with cardiovascular diseases. International journal of medical informatics 2017, 98:65-75.

57. Choi BG, Dhawan T, Metzger K, Marshall L, Akbar A, Jain T, Young HA, Katz RJ: Image-Based Mobile System for Dietary Management in an American Cardiology Population: Pilot Randomized Controlled Trial to Assess the Efficacy of Dietary Coaching Delivered via a Smartphone App Versus Traditional Counseling. JMIR mHealth and uHealth 2019, 7(4):e10755.

58. Hansel B, Giral P, Gambotti L, Lafourcade A, Peres G, Filipecki C, Kadouch D, Hartemann A, Oppert J-M, Bruckert E: A fully automated web-based program improves lifestyle habits and HbA1c in patients with type 2 diabetes and abdominal obesity: Randomized trial of patient E-coaching nutritional support (The ANODE Study). Journal of medical Internet research 2017, 19(11):e360.

59. Lim S, Kang SM, Kim KM, Moon JH, Choi SH, Hwang H, Jung HS, Park KS, Ryu JO, Jang HC: Multifactorial intervention in diabetes care using real-time monitoring and tailored feedback in type 2 diabetes. Acta diabetologica 2016, 53(2):189-198.

60. Alonso-Domínguez R, García-Ortiz L, Patino-Alonso MC, Sánchez-Aguadero N, Gómez-Marcos MA, Recio-Rodríguez JI: Effectiveness of a multifactorial intervention in increasing adherence to the mediterranean diet among patients with diabetes mellitus type 2: a controlled and randomized study (EMID study). Nutrients 2019, 11(1):162.

61. Green BB, Anderson ML, Cook AJ, Catz S, Fishman PA, McClure JB, Reid RJ: eCare for heart wellness: a feasibility trial to decrease blood pressure and cardiovascular risk. Am J Prev Med 2014, 46(4):368-377.

62. Kelly JT, Conley M, Hoffmann T, Craig JC, Tong A, Reidlinger DP, Reeves MM, Howard K, Krishnasamy R, Kurtkoti J: A Coaching Program to Improve Dietary Intake of Patients with CKD: ENTICE-CKD. Clinical Journal of the American Society of Nephrology 2020, 15(3):330-340.

63. Castelnuovo G, Manzoni GM, Cuzziol P, Cesa GL, Corti S, Tuzzi C, Villa V, Liuzzi A, Petroni ML, Molinari E: TECNOB study: ad interim results of a randomized controlled trial of a multidisciplinary telecare intervention for obese patients with type-2 diabetes. Clinical practice and epidemiology in mental health: CP \& EMH 2011, 7:44.

64. Allen JK, Stephens J, Dennison Himmelfarb CR, Stewart KJ, Hauck S: Randomized controlled pilot study testing use of smartphone technology for obesity treatment. Journal of obesity 2013, 2013.

65. Hurkmans E, Matthys C, Bogaerts A, Scheys L, Devloo K, Seghers J: Face-toface versus mobile versus blended weight loss program: randomized clinical trial. JMIR mHealth and uHealth 2018, 6(1):e14. 
66. Turnin M, Bourgeois O, Cathelineau G, Leguerrier A, Halimi S, Sandre-Banon $D$, Coliche V, Breux M, Verlet E, Labrousse F: Multicenter randomized evaluation of a nutritional education software in obese patients. 2008.

67. Rader S, Dorner TE, Schoberberger R, Wolf H: Effects of a web-based follow-up intervention on self-efficacy in obesity treatment for women. Wiener klinische Wochenschrift 2017, 129(13-14):472-481.

68. Veazie S, Winchell K, Gilbert J, Paynter R, Ivlev I, Eden KB, Nussbaum K, Weiskopf N, Guise J-M, Helfand M: Rapid evidence review of mobile applications for self-management of diabetes. Journal of general internal medicine 2018, 33(7):1167-1176.

69. Pai A: Survey: 58 percent of smartphone users have downloaded a fitness or health app [https://www.mobihealthnews.com/48273/survey58-percent-of-smartphone-users-have-downloaded-a-fitness-or-health-app]

70. Nadarzynski T, Miles 0, Cowie A, Ridge D: Acceptability of artificial intelligence (AI)-led chatbot services in healthcare: A mixed-methods study. Digital health 2019, 5:2055207619871808.

71. Health Hero: Slack Health Hero [https://fa.ebotstore.com/bot/a02sjhzq9health-hero-slack]

72. Tasteful: Healthy Eating App, Tasteful, Gets Personal With New Chatbot Feature [https://www.globenewswire.com/newsrelease/2016/05/20/1126243/0/en/Healthy-Eating-App-Tasteful-GetsPersonal-With-New-Chatbot-Feature.html]

73. Lark Health: Lark Health Offers On-Demand Behavioral Health Coaching at No Cost to Help People Manage Stress \& Anxiety During the COVID-19 Pandemic [https://www.lark.com/]

74. Forksy Nutrition advice automation: Automate Nutrition Coaching with AI [https://getforksy.com/]

75. Laranjo L, Dunn AG, Tong HL, Kocaballi AB, Chen J, Bashir R, Surian D, Gallego B, Magrabi F, Lau AY: Conversational agents in healthcare: a systematic review. Journal of the American Medical Informatics Association 2018, 25(9):1248-1258.

76. Bickmore TW, Schulman D, Sidner C: Automated interventions for multiple health behaviors using conversational agents. Patient education and counseling 2013, 92(2):142-148.

77. Migneault JP, Dedier JJ, Wright JA, Heeren T, Campbell MK, Morisky DE, Rudd $\mathrm{P}$, Friedman RH: A culturally adapted telecommunication system to improve physical activity, diet quality, and medication adherence among hypertensive African-Americans: a randomized controlled trial. Annals of Behavioral Medicine 2012, 43(1):62-73.

78. Vo R, Smith M, Patton N: A model of the multidimensional nature of experienced dietitian clinical decision - making in the acute care setting. Journal of Human Nutrition and Dietetics 2020.

79. Chen J, Lieffers J, Bauman A, Hanning R, Allman - Farinelli M: The use of smartphone health apps and other mobile $h$ ealth (mHealth) technologies in dietetic practice: a three country study. Journal of Human Nutrition and Dietetics 2017, 30(4):439-452. 
80. Jospe MR, Fairbairn KA, Green P, Perry TL: Diet app use by sports dietitians: a survey in five countries. JMIR mHealth and uHealth 2015, 3(1):e7.

81. Lieffers JR, Vance VA, Hanning RM: Use of mobile device applications in Canadian dietetic practice. Canadian journal of dietetic practice and research 2014, 75(1):41-47.

82. Muehlhausen W, Doll H, Quadri N, Fordham B, O'Donohoe P, Dogar N, Wild DJ: Equivalence of electronic and paper administration of patientreported outcome measures: a systematic review and meta-analysis of studies conducted between 2007 and 2013. Health and quality of life outcomes 2015, 13(1):167.

83. Cella D HE, Jensen SE, et al,: Patient-Reported Outcomes in Performance Measurement. In.: Research Triangle Park (NC): RTI Press. Available from: https://www.ncbi.nlm.nih.gov/books/NBK424381/; 2015.

84. Academy of Nutrition and Dietetics: ANDHII [https://www.eatrightpro.org/research/projects-tools-andinitiatives/andhii]

85. Murphy WJ, Yadrick MM, Steiber AL, Mohan V, Papoutsakis C: Academy of Nutrition and Dietetics Health Informatics Infrastructure (ANDHII): A pilot study on the documentation of the Nutrition Care Process and the usability of ANDHII by registered dietitian nutritionists. Journal of the Academy of Nutrition and Dietetics 2018, 118(10):1966-1974.

86. Roberts S, Hopper Z, Chaboyer W, Gonzalez R, Banks M, Desbrow B, Marshall AP: Engaging hospitalised patients in their nutrition care using technology: development of the NUTRI-TEC intervention. $B M C$ health services research 2020, 20(1):1-11.

87. ORCHA: Release the benefits of digital health for your patients \& populations [https://www.orcha.co.uk/who-we-help/health-careprofessionals/]

88. NHS: NHS Apps Library [https://www.nhs.uk/apps-library/]

89. Academy of Nutrition and Dietetics: Practicing Telehealth [https://www.eatrightpro.org/practice/practiceresources/telehealth/practicing-telehealth]

90. Practice-based Evidence in Nutrition (PEN): Telehealth/Teledietetics [https://www.pennutrition.com/KnowledgePathway.aspx?kpid=18478]

91. British Dietetic Association: BDA statement on avoidable contact and use of technological solutions during COVID-19 pandemic [https://www.bda.uk.com/resource/bda-statement-on-avoidable-contactand-use-of-technological-solutions-during-covid-19-pandemic.html]

92. Australia DAo: National competency standards for dietitians in Australia. 2015.

93. Education PfD, Practice: The Integrated Competencies for Dietetic Education and Practice. 2012.

94. Association BD: A curriculum framework for the pre-registration education and training of dietitians 2013. In.; 2018. 
95. Rollo ME, Collins CE, MacDonald-Wicks L: Evaluation of the introduction of an e-health skills component for dietetics students. Telemed E-Health 2017, 23(11):930-933.

96. Nebeker C, Murray K, Holub C, Haughton J, Arredondo EM: Acceptance of mobile health in communities underrepresented in biomedical research: barriers and ethical considerations for scientists. JMIR mHealth and uHealth 2017, 5(6):e87.

97. Research and Markets: Global Internet of Things Policies 2018-2019 [https://www.globenewswire.com/newsrelease/2019/05/30/1859482/0/en/Global-Internet-of-Things-Policies2018-2019.html]

98. Islam SR, Kwak D, Kabir MH, Hossain M, Kwak K-S: The internet of things for health care: a comprehensive survey. IEEE Access 2015, 3:678-708.

99. Board NI: Personalised health and care 2020: using data and technology to transform outcomes for patients and citizens: a framework for action. In.: HM Government London; 2014.

100. Asthana S, Jones R, Sheaff R: Why does the NHS struggle to adopt eHealth innovations? A review of macro, meso and micro factors. $B M C$ health services research 2019, 19(1):1-7.

101. eHealth WGOf: Global Diffusion of EHealth: Making Universal Health Coverage Achievable: Report of the Third Global Survey on EHealth: World Health Organization; 2016.

102. World Health Organization: Directory of eHealth policies [https://www.who.int/goe/policies/countries/en/]

103. Pavlovic Y: The US Food and Drug Administration regulation of digital health. In: Diabetes Digital Health. edn.: Elsevier; 2020: 253-269.

104. Food \& Drug Administration: Device Software Functions Including Mobile Medical Applications [https://www.fda.gov/medical-devices/digitalhealth/device-software-functions-including-mobile-medical-applications]

105. Australian Digital Health Agency: For Developers [https://developer.digitalhealth.gov.au/]

106. Pustozerov E, Albrecht U: Evaluation of mHealth applications security based on application permissions. Studies in health technology and informatics 2016, 226:241-244.

107. Rubí JNS, Gondim PRdL: Interoperable Internet of Medical Things platform for e-Health applications. International Journal of Distributed Sensor Networks 2020, 16(1):1550147719889591.

108. Mandel JC, Kreda DA, Mandl KD, Kohane IS, Ramoni RB: SMART on FHIR: a standards-based, interoperable apps platform for electronic health records. Journal of the American Medical Informatics Association 2016, 23(5):899-908.

109. Lehne M, Luijten S, IMBUSCH PVFG, Thun S: The Use of FHIR in Digital Health-A Review of the Scientific Literature. Studies in health technology and informatics 2019, 267:52-58. 
110. Academy of Nutrition and Dietetics: Telehealth and Payment [https://www.eatrightpro.org/practice/practiceresources/telehealth/telehealth-payment]

111. Knotowicz H, Haas A, Coe S, Furuta GT, Mehta P: Opportunities for Innovation and Improved Care Using Telehealth for Nutritional Interventions. Gastroenterology 2019, 157(3):594-597.

112. Department of Health: COVID-19: Whole of population telehealth for patients, general practice, primary care and other medical services: A joint media release with Professor Michael Kidd AM, Principle Medical Advisor [https://www.health.gov.au/ministers/the-hon-greg-hunt$\mathrm{mp} / \mathrm{media} /$ covid-19-whole-of-population-telehealth-for-patients-generalpractice-primary-care-and-other-medical-services]

113. CMS.gov: Medicare telemedicine health care provider fact sheet [https://www.cms.gov/newsroom/fact-sheets/medicare-telemedicinehealth-care-provider-fact-sheet]

114. Smith AC, Thomas E, Snoswell CL, Haydon H, Mehrotra A, Clemensen J, Caffery LJ: Telehealth for global emergencies: Implications for coronavirus disease 2019 (COVID-19). Journal of telemedicine and telecare 2020:1357633X20916567.

115. Webster P: Virtual health care in the era of COVID-19. The Lancet 2020, 395(10231):1180-1181.

116. Royal Australian College of General Practitioners: HANDI: Handbook of Non-Drug Interventions (HANDI) [https://www.racgp.org.au/clinicalresources/clinical-guidelines/handi]

117. Nutritools: Nutritools website. In.: Online. Accessed 20/06/2020. Available from: https://www.nutritools.org/; 2018.

118. American Psychiatric Association: The App Evaluation Model [https://www.psychiatry.org/psychiatrists/practice/mental-healthapps/app-evaluation-model-v2] 


\section{List of Tables}

Table 1: Selected mHealth databases, resources and frameworks to evaluate and implement evidence-based digital care 


\section{List of Figures}

Figure 1: Digital technologies to compliment the Nutrition Care Process. Each '!' on the peripheral represents barriers to address for wider adoption in primary and secondary care. Abbreviations: BP: blood pressure, ECG: electrocardiogram, PREM: patient-reported experience measures, PROM: patient-reported outcome measures. 